\title{
Social Preferences under Risk: Equality of Opportunity versus Equality of Outcome
}

\author{
By Kota SAITo*
}

$X$ and $Y$ are brothers who like to share everything, whenever possible. They are also both huge fans of football. One day, $X$ finds an envelope in the park that contains just one ticket to the next Super Bowl. $X$ agonizes over whether to (i) attend the game secretly, which would make him feel guilty; or (ii) tell his brother about it and suggest they flip a coin to decide who gets to go. From an ex ante viewpoint, flipping a coin means that the brothers' chances of getting the ticket will be equal. From an ex post viewpoint, however, if $X$ loses the coin toss, he will definitely feel envy.

This parable shows that an individual could have a preference for two distinct notions of equality: equality of opportunity (i.e., equality of ex ante expected payoffs) and equality of outcome (i.e., equality of ex post payoffs). Moreover, the individual could face a trade-off between the two notions of equality.

In the literature on social preferences, many theories have been proposed on the inequality aversion of an individual, but most of them study deterministic environments. (See Camerer 2003 and Fehr and Schmidt 2006 for surveys.) However, it is not obvious how to appropriately extend such preferences to risky environments. As Fudenberg and Levine (2012, p. 606) point out, "leading theories of outcome-based social preferences fail to reflect concerns for ex ante fairness when they are extended to lotteries using expected utility theory as is implicit in some of this work."

In the social choice literature, several papers, including Diamond (1967), Machina (1989), and Gajdos and Maurin (2004), have studied the trade-off between the two notions of equality. However, they all focus on an impartial social planner. Much less attention has been devoted to studying a partially self-interested individual, who

\footnotetext{
* Division of the Humanities and Social Sciences, California Institute of Technology, MC 228-77 1200 E. California Blvd., Pasadena, CA 91125 (e-mail: saito@caltech.edu). I am indebted to my adviser, Eddie Dekel, for continuous guidance, support, and encouragement. I am grateful to Colin Camerer, Federico Echenique, Jeff Ely, Ernst Fehr, Faruk Gul, Pietro Ortoleva, Marciano Siniscalchi, and Leeat Yariv for discussions that have led to improvement of the paper. I would like to thank my former adviser, Michihiro Kandori, for his guidance when I was at the University of Tokyo. I would also like to thank Elchanan Ben-Porath, Kenneth Binmore, Soo Hong Chew, David Dillenberger, Thibault Gajdos, Itzhak Gilboa, Shachar Kariv, Edi Karni, David Levine, Fabio Maccheroni, Massimo Marinacci, Philippe Mongin, John Roemer, Jacob Sagi, Uzi Segal, and seminar participants at the University of Tokyo, Northwestern University, California Institute of Technology, and Risk, Uncertainty, and Decision (RUD 2010) in Paris. I gratefully acknowledge financial support from the Center for Economic Theory at the Economics Department of Northwestern University. Finally, I appreciate three anonymous referees for their comments. The author has no financial or other material interests related to this research to disclose. This paper was originally circulated as K. Saito (January 25, 2008) "Social Preference under Uncertainty," the University of Tokyo, COE Discussion Papers, No. F-217 (http://www2.e.u-tokyo.ac.jp/cemano/ research/DP/dp.html). The original paper on inequality aversion was first presented on January 16, 2007 at a micro-workshop seminar at the University of Tokyo.

${ }^{\dagger}$ Go to http://dx.doi.org/10.1257/aer.103.7.3084 to visit the article page for additional materials and author disclosure statement(s).
} 


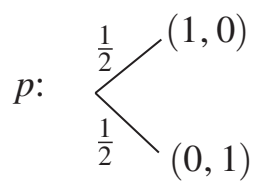

Figure 1. The Fifty-Fifty Lottery ( 1 denotes getting the ticket and 0 denotes not getting it)

might feel envy or guilt, although this seems more germane to recent experimental evidence that shows a significance of equality of opportunity. (In this article, the terms guilt and envy refer to the utility losses from advantageous inequality and disadvantageous inequality, respectively.)

For instance, in experiments on probabilistic dictator games, subjects are asked to allocate probabilities of winning an indivisible good with a passive recipient. In such experiments, Karni, Salmon, and Sopher (2008); Bohnet et al. (2008); Kircher, Ludwig, and Sandroni (2013); and Bolton and Ockenfels (2010) found that a considerable number of subjects chose a probability of winning the good equally.

The purpose of this paper is to provide a step toward filling this gap. We axiomatize a model of an individual's inequality aversion under risk that captures a preference for equality of opportunity and equality of outcome. We call this model the expected inequality-averse (EIA) model. In the EIA model, a unique number captures an individual's preference for equality of opportunity relative to equality of outcome. This parameter determines how the individual behaves when he faces the trade-off between the two notions of equality.

The rest of this article is organized as follows. In Section I, we define the EIA model and show its uniqueness. In Section II, we provide an axiomatic foundation for the EIA model and a characterization of the parameters. In Section III, we show that the EIA model is consistent with the recent experiments on probabilistic dictator games. In Section IV, we discuss recent related literature, namely, Fudenberg and Levine (2012) and Borah (2011). Finally, in Section V, we discuss an extension of the EIA model. All proofs are in the Appendix.

\section{The EIA Model}

We assume that the set of payoffs is $\mathbb{R}$. Let $I=\{1, \ldots, n\}$ be the set of individuals and let 1 denote the decision maker. A vector $\mathbf{x}=\left(x_{1}, \ldots, x_{n}\right)$ in $\mathbb{R}^{n}$ is called an allocation of payoffs among the individuals. A probability distribution $p$ on $\mathbb{R}^{n}$ with finite support is called a lottery. The set of all lotteries is denoted by $\Delta\left(\mathbb{R}^{n}\right)$. The primitive of our model is a binary relation $\succsim$ on $\Delta\left(\mathbb{R}^{n}\right)$ that describes the preference of the decision maker (i.e., agent $1 \in I$ ). As usual, $\succ$ and $\sim$ denote the asymmetric and symmetric parts of $\succsim$, respectively. The following five axioms characterize the EIA model. The first axiom is standard.

For any $p \in \Delta\left(\mathbb{R}^{n}\right), E_{p}$ is the expectation operator with respect to $p$. That is, if $p$ is a lottery that yields allocation $\mathbf{x}^{s}=\left(x_{1}^{s}, \ldots, x_{n}^{s}\right)$ with probability $\lambda_{s}$ for each $s \in\{1, \ldots, m\}$, then $E_{p}(\mathbf{x})=\sum_{s=1}^{m} \lambda_{s} \mathbf{x}^{s}=\left(\sum_{s=1}^{m} \lambda_{s} x_{1}^{s}, \ldots, \sum_{s=1}^{m} \lambda_{s} x_{n}^{s}\right)$. In addition, for any real-valued function $U$ on $\mathbb{R}^{n}, E_{p}(U(\mathbf{x}))=\sum_{s=1}^{m} \lambda_{s} U\left(\mathbf{x}^{s}\right)$. 
Definition: An expected inequality-averse $(E I A)$ model is a triple $(\alpha, \beta, \delta) \in \mathbb{R}_{+}^{n-1}$ $\times \mathbb{R}_{+}^{n-1} \times[0,1]$ such that $\succsim$ is represented by the function $V$ on $\Delta\left(\mathbb{R}^{n}\right)$ defined by

$$
V(p)=\delta U\left(E_{p}(\mathbf{x})\right)+(1-\delta) E_{p}(U(\mathbf{x})),
$$

where $U(\mathbf{x})=x_{1}-\sum_{i=2}^{n}\left(\alpha_{i} \max \left\{x_{i}-x_{1}, 0\right\}+\beta_{i} \max \left\{x_{1}-x_{i}, 0\right\}\right)$.

The function $U$ corresponds to the model proposed by Fehr and Schmidt (1999) and captures inequality aversion as follows: for each $i \neq 1$, the term $\alpha_{i} \max \left\{x_{i}-x_{1}, 0\right\}$ captures the disutility of envy when the decision maker's payoff $x_{1}$ is smaller than individual $i$ 's payoff $x_{i}$; the term $\beta_{i} \max \left\{x_{1}-x_{i}, 0\right\}$ captures the disutility of guilt when the decision maker's payoff $x_{1}$ is larger than individual $i$ 's payoff $x_{i}$.

With this function $U$, the EIA model captures preferences for the two notions of equality as follows. The term $U\left(E_{p}(\mathbf{x})\right)$ represents a preference for equality of opportunity because it captures inequality aversion in the ex ante expected allocations (i.e., $\left.E_{p}(\mathbf{x})\right)$. In contrast, the term $E_{p}(U(\mathbf{x}))$ represents a preference for equality of outcome because it captures expected inequality aversion in the ex post allocations (i.e., $\mathbf{x}$ ). Therefore, the weight $\delta$ on the first term captures a preference for equality of opportunity relative to equality of outcome.

Remark: Suppose that two EIA models $(\alpha, \beta, \delta)$ and $\left(\alpha^{\prime}, \beta^{\prime}, \delta^{\prime}\right)$ represent $\succsim$. Then, (i) $(\alpha, \beta)=\left(\alpha^{\prime}, \beta^{\prime}\right)$ and $($ ii $)$ if $(\alpha, \beta) \neq \mathbf{0} \neq\left(\alpha^{\prime}, \beta^{\prime}\right), \delta=\delta^{\prime}$.

In the remark, result (i) means that $\alpha$ and $\beta$ are uniquely pinned down for the decision maker. Result (ii) means that $\delta$ is also uniquely pinned down when the decision maker is inequality averse (i.e., when $(\alpha, \beta) \neq \mathbf{0})$. When the decision maker is not inequality averse (i.e., $(\alpha, \beta)=\mathbf{0})$, his utility equals to his expected payoff (i.e., $\left.V(p)=E_{p}\left(x_{1}\right)\right)$ because $U\left(E_{p}(\mathbf{x})\right)=E_{p}\left(x_{1}\right)=E_{p}(U(\mathbf{x}))$. Hence, $\delta$ becomes irrelevant to the decision maker's utility.

\section{Axioms}

Axiom (Rationality): $\succsim$ is complete, transitive, continuous, and monotonic in equal allocations (i.e., $(x, \ldots, x) \succsim(y, \ldots, y)$ if and only if $x \geq y)$. $^{1}$

In the next axiom, conditions (i) and (ii) capture envy and guilt, in that order. In the axiom, $\left(x,(y)_{-i}\right)$ denotes allocation that gives payoff $x$ only to individual $i$ and gives payoff $y$ to all the other individuals. Remember that $1 \in I$ denotes the decision maker.

Axiom (Inequality Aversion): For all $i \neq 1$, (i) $\left(0,(0)_{-i}\right) \succsim\left(1,(0)_{-i}\right)$ and (ii) $\left(0,(0)_{-i}\right) \succsim\left(-1,(0)_{-i}\right)$.

Since these conditions are required only for $i \neq 1$, this axiom allows the standard preference that $\mathbf{x} \succsim \mathbf{y}$ if and only if $x_{1} \geq y_{1}$.

\footnotetext{
${ }^{1}$ The continuity assumed here is von Neumann-Morgenstern continuity with respect to outcome mixtures and probability mixtures. Formally, (i) for all $\mathbf{x}, \mathbf{y}, \mathbf{z} \in \mathbb{R}^{n}$, if $\mathbf{x} \succ \mathbf{y}$ and $\mathbf{y} \succ \mathbf{z}$, then there exist $\lambda$ and $\mu$ in $(0,1)$ such that $\lambda \mathbf{x}+(1-\lambda) \mathbf{z} \succ \mathbf{y}$ and $\mathbf{y} \succ \mu \mathbf{x}+(1-\mu) \mathbf{z}$; and (ii) for all $p, q, r \in \Delta\left(\mathbb{R}^{n}\right)$, if $p \succ q$ and $q \succ r$, then there exist $\lambda$ and $\mu$ in $(0,1)$ such that $\lambda p \oplus(1-\lambda) r \succ q$ and $q \succ \mu p \oplus(1-\mu) r$.
} 
To provide a weaker version of the independence axiom, we first define an outcome mixture of allocations as follows:

Definition: For all $\lambda \in[0,1]$ and $\mathbf{x}, \mathbf{y} \in \mathbb{R}^{n}, \lambda \mathbf{x}+(1-\lambda) \mathbf{y} \in \mathbb{R}^{n}$ is an allocation such that $\lambda \mathbf{x}+(1-\lambda) \mathbf{y}=\left(\lambda x_{1}+(1-\lambda) y_{1}, \ldots, \lambda x_{n}+(1-\lambda) y_{n}\right)$.

Inequality-averse preferences may violate the independence axiom with respect to outcome mixtures. For example, in (two-agent) deterministic dictator games, a significant number of subjects prefer allocating an endowment equally rather than consuming the whole endowment by themselves (i.e., $\left.\left(\frac{1}{2}, \frac{1}{2}\right) \succ(1,0)\right)$. However, the same subjects often prefer consuming the whole endowment by themselves rather than giving it away to the receiver (i.e., $(1,0) \succ(0,1))$. It follows that $\left(\frac{1}{2}, \frac{1}{2}\right) \succ(1,0) \succ(0,1)$ but $\frac{1}{2}(1,0)+\frac{1}{2}(0,1) \equiv\left(\frac{1}{2}, \frac{1}{2}\right)$, which violates the independence axiom. ${ }^{2}$

The source of the violation here is the fact that in the mixed allocations, the rankings of the outcomes are opposite (i.e., the other individual is better off than the decision maker in $(0,1)$ and worse off in $(1,0))$. We want to assume the independence axiom only when the mixture does not involve such opposite outcomes.

Definition: Two allocations $\mathbf{x}, \mathbf{y} \in \mathbb{R}^{n}$ are said to be quasi-comonotonic if there is no $i \neq 1$ such that $x_{i}>x_{1}$ and $y_{i}<y_{1}$.

Note that if $\mathbf{x}$ and $\mathbf{y}$ are quasi-comonotonic, then the rankings of the payoffs of any individual with respect to the decision maker are not opposite between $\mathbf{x}$ and $\mathbf{y} \cdot{ }^{3}$

Axiom (Quasi-Comonotonic Independence for Outcome Mixture): For all $\lambda \in(0,1]$ and $\mathbf{x}, \mathbf{y}, \mathbf{z} \in \mathbb{R}^{n}$ such that the pairs $\mathbf{x}, \mathbf{z}$, and $\mathbf{y}, \mathbf{z}$ are each quasi-comonotonic,

$$
\mathbf{x} \succsim \mathbf{y} \Leftrightarrow \lambda \mathbf{x}+(1-\lambda) \mathbf{z} \succsim \lambda \mathbf{y}+(1-\lambda) \mathbf{z}
$$

These three axioms characterize Fehr and Schmidt's (1999) model on $\mathbb{R}^{n}$ (i.e., the set of degenerate lotteries on allocations). (See Lemma 1 in the Appendix for the formal statement.)

The next axiom is a weaker version of the independence axiom with respect to probability mixtures. Before stating the axiom, we formally define probability mixtures by using the notation $\oplus$ :

Definition: For all $\lambda \in[0,1]$ and $p, q \in \Delta\left(\mathbb{R}^{n}\right), \lambda p \oplus(1-\lambda) q \in \Delta\left(\mathbb{R}^{n}\right)$ is a lot tery such that $(\lambda p \oplus(1-\lambda) q)(\mathbf{x})=\lambda p(\mathbf{x})+(1-\lambda) q(\mathbf{x}) \in[0,1]$ for each $\mathbf{x} \in \mathbb{R}^{n} .4$

\footnotetext{
${ }^{2}$ Note that mixtures here are not probability mixtures.

${ }^{3}$ The definition is a weaker version of comonotonicity provided by Schmeidler (1989). Schmeidler (1989) presents an interpretation of comonotonicity from the viewpoint of a social planner's inequality aversion, as follows: two allocations $\mathbf{x}$ and $\mathbf{y}$ are comonotonic if the social rank of any two individuals is not reversed between $\mathbf{x}$ and $\mathbf{y}$. When we focus on an individual's inequality-averse preferences, what is relevant to the individual is social rank with respect to the individual himself, not the social rank of any two individuals.

${ }^{4}$ For example, lottery $p$ in Figure 1 corresponds to probability mixture $\frac{1}{2}(1,0) \oplus \frac{1}{2}(0,1)$. For simplicity, instead of writing $\lambda \delta_{\mathbf{x}} \oplus(1-\lambda) \delta_{\mathbf{y}}$, we write $\lambda \mathbf{x} \oplus(1-\lambda) \mathbf{y}$, where $\delta_{\mathbf{x}}$ and $\delta_{\mathbf{y}}$ denote the degenerate lotteries that yield $\mathbf{x}$ and $\mathbf{y}$ with probability 1 , respectively.
} 
Just as outcome mixtures may change the rankings of ex post payoffs, probability mixtures may change the rankings of ex ante expected payoffs. However, probability mixtures with an equal allocation $(x, \ldots, x)$ should not change any such rankings. This suggests the following axiom:

Axiom (Constant Independence for Probability Mixture): For all $\lambda \in(0,1]$, $p, q \in \Delta\left(\mathbb{R}^{n}\right)$, and $x \in \mathbb{R}$,

$$
p \succsim q \Leftrightarrow \lambda p \oplus(1-\lambda)(x, \ldots, x) \succsim \lambda q \oplus(1-\lambda)(x, \ldots, x) .
$$

Under the above four axioms, for any allocation $\mathbf{x}$, it will be shown that there exists a unique real number $z$ such that $(z, \ldots, z) \sim \mathbf{x}$. Such a payoff $z$ is denoted by $e(\mathbf{x})$ and is called an equality equivalent. The value $e(\mathbf{x})$ reflects the equality of an allocation $\mathbf{x}$.

The final key axiom, dominance, states that if a lottery $p$ is superior to a lottery $q$ in regard to both equality of opportunity and equality of outcome, then $p$ should be preferred to $q$. To show the axiom, we formalize the two ways to evaluate equality of a lottery based on the two notions of equality. Consider a lottery $p=\lambda_{1} \mathbf{x}^{1} \oplus \cdots \oplus \lambda_{m} \mathbf{x}^{m}$. Then, $E_{p}(\mathbf{x})=\left(\sum_{s=1}^{m} \lambda_{s} x_{1}^{s}, \ldots, \sum_{s=1}^{m} \lambda_{s} x_{n}^{s}\right)$ is the expected allocation in $p$. Hence, $e\left(E_{p}(\mathbf{x})\right)$ reflects the evaluation of $p$ in terms of equality of opportunity. In contrast, since each $e\left(\mathbf{x}^{s}\right)$ reflects the equality of outcome $\mathbf{x}^{s}, E_{p}(e(\mathbf{x}))=\sum_{s=1}^{m} \lambda_{s} e\left(\mathbf{x}^{s}\right)$ is the expected evaluation of $p$ in terms of equality of outcome. By using these two formalizations, we can state the axiom as follows:

Axiom (Dominance): For all $p, q \in \Delta\left(\mathbb{R}^{n}\right)$,

$$
\left\{\begin{array}{l}
\text { (i) } e\left(E_{p}(\mathbf{x})\right) \geq e\left(E_{q}(\mathbf{x})\right) \\
\text { (ii) } E_{p}(e(\mathbf{x})) \geq E_{q}(e(\mathbf{x}))
\end{array}\right) \Rightarrow p \succsim q .
$$

To demonstrate how to use the dominance axiom, consider two lotteries $p=\frac{1}{2}(1,1) \oplus \frac{1}{2}(0,0)$ and $q=\frac{1}{2}(1,0) \oplus \frac{1}{2}(0,1)$. Suppose that the decision maker is strictly inequality averse (i.e., $(1,1) \succ(1,0)$ and $(0,0) \succ(0,1))$. On the one hand, $E_{p}(\mathbf{x})=\left(\frac{1}{2}, \frac{1}{2}\right)=E_{q}(\mathbf{x})$, so that $e\left(E_{p}(\mathbf{x})\right)=\frac{1}{2}=e\left(E_{q}(\mathbf{x})\right)$. Hence, $p$ and $q$ are equally desirable in regard to equality of opportunity.

On the other hand, by the strict inequality aversion, $1>e(1,0)$ and $0>e(0,1)$. Since $e(1,1)=1$ and $e(0,0)=0$, it follows that $E_{p}(e(\mathbf{x}))=\frac{1}{2} e(1,1)+\frac{1}{2} e(0,0)$ $=\frac{1}{2}>\frac{1}{2} e(1,0)+\frac{1}{2} e(0,1)=E_{q}(e(\mathbf{x}))$. Hence, $p$ is preferred to $q$ in regard to equality of outcome. Therefore, the dominance axiom implies that $p$ is at least as good as $q \cdot{ }^{5}$

\footnotetext{
${ }^{5}$ We do not require a strict version of the dominance axiom (i.e., if one of the inequalities in (i) and (ii) is strict then the preference should be strict). Indeed, the strict version does not allow two special EIA models where $\delta$ is either zero or one.
} 
The above five axioms are equivalent to an EIA model. Formally,
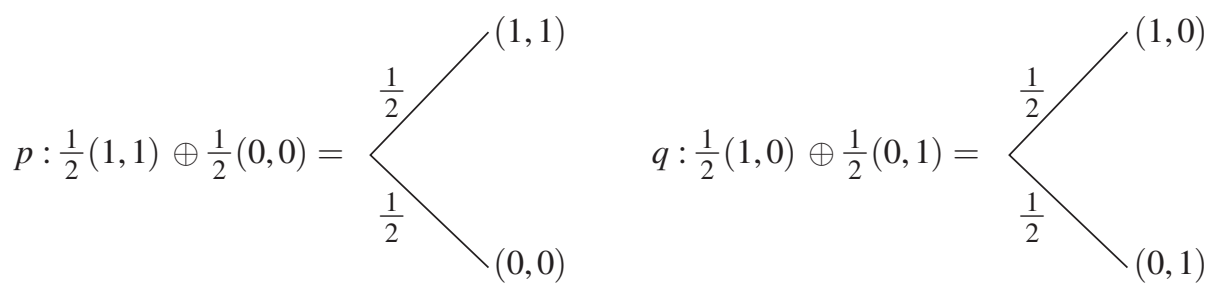

(i) $e\left(E_{p}(\mathbf{x})\right)=e\left(\frac{1}{2}, \frac{1}{2}\right)$

$$
\begin{aligned}
& e\left(E_{q}(\mathbf{x})\right)=e\left(\frac{1}{2}, \frac{1}{2}\right) \\
& E_{q}(e(\mathbf{x}))=\frac{1}{2} e(1,0)+\frac{1}{2} e(0,1)
\end{aligned}
$$$$
\text { (ii) } E_{p}(e(\mathbf{x}))=\frac{1}{2} e(1,1)+\frac{1}{2} e(0,0)
$$

Figure 2. Dominance

THEOREM: $\succsim$ satisfies Rationality, Inequality Aversion, Quasi-Comonotonic Independence for Outcome Mixture, Constant Independence for Probability Mixture, and Dominance if and only if there exists an EIA model $(\alpha, \beta, \delta)$.

\section{A. Parameter Characterization}

We can identify the parameters $\alpha, \beta$, and $\delta$ easily by experiments. We can identify $\alpha$ and $\beta$ by eliciting real numbers $\alpha_{i}$ and $\beta_{i}$ such that

$$
\left(-\alpha_{i}, \ldots,-\alpha_{i}\right) \sim\left(1,(0)_{-i}\right) \text { and }\left(-\beta_{i}, \ldots,-\beta_{i}\right) \sim\left(-1,(0)_{-i}\right)
$$

for each $i \in I \backslash\{1\} .6$ (Here, we use 1 and -1 just for simplicity. Instead of 1 and -1 , we could use any real numbers $x$ and $y$ such that $x>y$, which would be more appropriate for experiments. $)^{7}$

After identifying $\alpha$ and $\beta$, we can identify $\delta$ by eliciting a real number $c$ such that

$$
(c, \ldots, c) \sim \frac{1}{2}\left(1,(0)_{-i}\right) \oplus \frac{1}{2}\left(-1,(0)_{-i}\right),
$$

for some $i \in I \backslash\{1\}$. Then, the EIA model shows that $c=-\frac{1}{2}(1-\delta)\left(\alpha_{i}+\beta_{i}\right)$. Hence, $\delta=1+\frac{2 c}{\alpha_{i}+\beta_{i}} .8$

In what follows, we characterize the special cases of the EIA model in which $\delta=1$ and $\delta=0$. The next axiom characterizes the special case of $\delta=1$. The axiom means that the decision maker cares only about the expected payoffs.

\footnotetext{
${ }^{6}$ To see that $\alpha_{i}$ and $\beta_{i}$ are nonnegative, note that by inequality aversion, $\left(0,(0)_{-i}\right) \succsim\left(1,(0)_{-i}\right)$ and $\left(0,(0)_{-i}\right) \succsim$ $\left(-1,(0)_{-i}\right)$ for each $i \in I \backslash\{1\}$. Hence, by monotonicity, $\alpha_{i}$ and $\beta_{i}$ are nonnegative.

${ }^{7}$ In that case, $\frac{x+y}{2}$ must be used instead of zero.

${ }^{8}$ To see that $\delta$ can be smaller than one, note that for inequality-averse subjects, it is natural to assume that $\left(0,(0)_{-i}\right) \succsim \frac{1}{2}\left(1,(0)_{-i}\right) \oplus \frac{1}{2}\left(-1,(0)_{-i}\right)$. Hence, under monotonicity, $c$ is negative. Thus, $\delta \leq 1$.
} 
Axiom (Indifference to Timing of Mixture): For all $i \neq 1$,

$$
(0, \ldots, 0) \sim \frac{1}{2}\left(1,(0)_{-i}\right) \oplus \frac{1}{2}\left(-1,(0)_{-i}\right) .
$$

The other special case of $\delta=0$ has been used in several papers such as Fehr, Klein, and Schmidt (2007) and Grund and Sliwka (2005). This special case is characterized by the axiom that states the decision maker is indifferent to probability mixtures.

Axiom (Indifference to Probability Mixture): For all $i \neq 1$ and $x, y \in \mathbb{R}$ such that $x>0>y$,

$$
\left(x,(0)_{-i}\right) \sim\left(y,(0)_{-i}\right) \Rightarrow \frac{1}{2}\left(x,(0)_{-i}\right) \oplus \frac{1}{2}\left(y,(0)_{-i}\right) \sim\left(x,(0)_{-i}\right) .
$$

PROPOSITION 1: For any EIA model $(\alpha, \beta, \delta)$ if $(\alpha, \beta) \neq \mathbf{0}$ then, the following holds:

(i) $\succsim$ exhibits indifference to timing of mixture if and only if $\delta=1$.

(ii) $\succsim$ exhibits indifference to probability mixture if and only if $\delta=0$.

\section{PROOF:}

Assume $\alpha_{i}>0$ for some $i \neq 1$, without loss of generality. To prove (i), note that $V\left(\frac{1}{2}\left(1,(0)_{-i}\right) \oplus \frac{1}{2}\left(-1,(0)_{-i}\right)\right)=-\frac{1}{2}(1-\delta)\left(\alpha_{i}+\beta_{i}\right)$. Hence, $\left(0,(0)_{-i}\right) \sim$ $\frac{1}{2}\left(1,(0)_{-i}\right) \oplus \frac{1}{2}\left(-1,(0)_{-i}\right) \Leftrightarrow \delta=1$, so that $(\mathrm{i})$ holds.

To prove (ii), choose any $x, y \in \mathbb{R}$ such that $x \succ 0 \succ y$ and $\left(x,(0)_{-i}\right) \sim\left(y,(0)_{-i}\right)$. Then, $U\left(x,(0)_{-i}\right)=-\alpha_{i} x$ and $U\left(y,(0)_{-i}\right)=\beta_{i} y$. Consider the case where $\frac{1}{2} x+$ $\frac{1}{2} y \geq 0$. In this case, $V\left(\frac{1}{2}\left(x,(0)_{-i}\right) \oplus \frac{1}{2}\left(y,(0)_{-i}\right)\right)=-\delta \alpha_{i}\left[\frac{1}{2} x+\frac{1}{2} y\right]-(1-\delta)$ $\times \frac{1}{2}\left[\alpha_{i} x-\beta_{i} y\right]$. Then $V\left(\frac{1}{2}\left(x,(0)_{-i}\right) \oplus \frac{1}{2}\left(y,(0)_{-i}\right)\right)-U\left(x,(0)_{-i}\right)=\delta \alpha_{i} \frac{1}{2}(x-y)$. Hence, $\frac{1}{2}\left(x,(0)_{-i}\right) \oplus \frac{1}{2}\left(y,(0)_{-i}\right) \sim\left(x,(0)_{-i}\right) \Leftrightarrow \delta=0$. For the other case where $\frac{1}{2} x+\frac{1}{2} y \leq 0$, the result can be proved in the same way.

The above proposition shows that the two special cases have rather extreme implications for behavior. This would suggest that most of the subjects would exhibit the generic case where $\delta \in(0,1)$.

\section{Consistency with Experiments}

In this section, we show that the EIA model can describe the experimental results in the probabilistic dictator games based on a tractable condition on the parameters (i.e., $\alpha, \beta$, and $\delta$ ). We investigate the probabilistic dictator game because this game provides a direct way to elicit preferences on lotteries over allocations. In addition, the game captures the essence of our parable of two brothers in the introduction.

In the probabilistic dictator games, a common experimental observation is that a substantial fraction of dictators share the probability of winning an indivisible good. For example, Kircher, Ludwig, and Sandroni (2013) asked 60 subjects to choose 
among receiving one euro, donating one euro to the Red Cross, and randomizing between these two options with equal probability. Kircher, Ludwig, and Sandroni (2013) found that 25 percent of the subjects chose the equal randomization. See Karni, Salmon, and Sopher (2008); Bohnet et al. (2008); and Bolton and Ockenfels (2010) for similar experimental evidence.

To see that this experimental finding is consistent with the EIA model, consider a dictator whose preference is represented by the EIA model. The dictator is asked to decide a probability $\lambda \in[0,1]$ that he will receive the indivisible good ex post. Since the recipient receives the good with the remaining probability $1-\lambda$, the dictator obtains a lottery $p^{\lambda} \equiv \lambda(1,0) \oplus(1-\lambda)(0,1) .{ }^{9}$ Thus, the utility obtained by choosing a probability $\lambda \in[0,1]$ is as follows: ${ }^{10}$

(1) $V\left(p^{\lambda}\right)= \begin{cases}\delta(\lambda-\alpha(1-2 \lambda))+(1-\delta)(\lambda(1+\alpha-\beta)-\alpha) & \text { if } \quad \lambda \leq \frac{1}{2}, \\ \delta(\lambda-\beta(2 \lambda-1))+(1-\delta)(\lambda(1+\alpha-\beta)-\alpha) & \text { if } \quad \lambda \geq \frac{1}{2} .\end{cases}$

Under the commonly used assumption that the disutility from envy is larger than that from guilt (i.e., $\alpha>\beta$ ), the optimal $\lambda^{*}$ (i.e., the maximizer of $(1)$ ) is characterized as follows:

PROPOSITION 2: Suppose that $\alpha>\beta$. The optimal probability is

$$
\lambda^{*}=\left\{\begin{array}{l}
\frac{1}{2} \quad \text { if } \quad \delta \geq \frac{1+\alpha-\beta}{\alpha+\beta}, \\
1 \quad \text { if } \delta \leq \frac{1+\alpha-\beta}{\alpha+\beta} .
\end{array}\right.
$$

PROOF:

Note that $\delta(\lambda-\alpha(1-2 \lambda))+(1-\delta)(\lambda(1+\alpha-\beta)-\alpha)-(\delta(\lambda-\beta(2 \lambda-1))+$ $(1-\delta)(\lambda(1+\alpha-\beta)-\alpha))=-\delta(\alpha-\beta)(1-2 \lambda)$. Since $\alpha>\beta$, therefore, $\lambda<\frac{1}{2}$ is not optimal.

For any $\lambda \in\left[\frac{1}{2}, 1\right]$, define $f(\lambda) \equiv V\left(p^{\lambda}\right)=(\alpha+\beta) \delta-\alpha+(1+\alpha-\beta-$ $\delta(\alpha+\beta)) \lambda$. By the linearity, $f$ attains the maximum at $\lambda=\frac{1}{2}$ or $\lambda=1$. Moreover, $f\left(\frac{1}{2}\right)>f(1) \Leftrightarrow \frac{1}{2}[1-(1-\delta)(\alpha+\beta)]>1-\beta \Leftrightarrow \delta>\frac{1+\alpha-\beta}{\alpha+\beta}$.

Therefore, the dictator shares the probability of winning the good if and only if $\delta \geq \frac{1+\alpha-\beta}{\alpha+\beta}$. Note the similarity between the probabilistic dictator game and the

\footnotetext{
${ }^{9}$ This specification of the allocation is without loss of generality. We can obtain the same result with $x$ and $y$ such that $x>y$, instead of one and zero. By the linearity of $V$ with respect to constant allocations, $V(\lambda(x, y) \oplus$ $(1-\lambda)(y, x))=V(\lambda(x-y, 0) \oplus(1-\lambda)(0, x-y))+V(y, y)=(x-y) V(\lambda(1,0) \oplus(1-\lambda)(0,1))+V(y, y)$. This calculation shows that the maximizer $\lambda$ for $V(\lambda(x, y) \oplus(1-\lambda)(y, x))$ must be the same as the maximizer $\lambda$ for $V\left(p^{\lambda}\right)$ for $x-y>0$.

${ }^{10}$ By definition, $V\left(p^{\lambda}\right)=\delta(\lambda-\alpha \max \{1-2 \lambda, 0\}-\beta \max \{2 \lambda-1,0\})+(1-\delta)(\lambda(1-\beta)+(1-\lambda)(-\alpha))$. A direct calculation shows the equation for each case.
} 
parable of the two brothers, in which $X$ agonizes over whether to (i) attend the game secretly or (ii) flip a coin to decide who gets to go. The result implies that $X$ should flip a coin (i.e., choose a lottery $\left.p=\frac{1}{2}(1,0) \oplus \frac{1}{2}(0,1)\right)$ if and only if $\delta>\frac{1+\alpha-\beta}{\alpha+\beta}$. Given the values of $\alpha$ and $\beta$ that are often assumed in the literature, this inequality condition is compatible with the restriction that $\delta \in[0,1] .11$

\section{Related Literature}

In this section, we discuss the recent related studies, Fudenberg and Levine (2012); Borah (2011); and Rohde (2010). Fudenberg and Levine (2012) investigate a twoagent and two-state model, in which each state realizes equally likely. In the setup, Fudenberg and Levine (2012) show two main results. Their first result shows that the independence axiom (with respect to probability mixtures) is incompatible with a preference for equality of opportunity. This result is consistent with our theorem because the EIA model captures a preference for equality of opportunity if and only if $\delta>0$ and $(\alpha, \beta) \neq \mathbf{0}$. Moreover, the EIA model also violates the independence axiom if and only if $\delta>0$ and $(\alpha, \beta) \neq \mathbf{0}$.

The second result in Fudenberg and Levine (2012) shows that there are no preferences that satisfy all of the following four properties: transitivity, a preference for equality of outcome, state independence, and their Property 5 (Correlation Invariance), which states that $\frac{1}{2}\left(x_{1}, x_{2}\right) \oplus \frac{1}{2}\left(y_{1}, y_{2}\right) \sim \frac{1}{2}\left(x_{1}, y_{2}\right) \oplus \frac{1}{2}\left(y_{1}, x_{2}\right)$. Our theorem is also consistent with this result as well, because any EIA model satisfies transitivity and state independence. (The state independence is satisfied trivially because we assume objective probability without state space.) In addition, the EIA model captures equality of outcome if and only if $\delta<1$ and $(\alpha, \beta) \neq \mathbf{0}$. Moreover, the EIA model violates Property 5 if and only if $\delta<1$ and $(\alpha, \beta) \neq \mathbf{0}$. To see this latter equivalence, note that Property 5 implies the indifference between the two lotteries $p \equiv \frac{1}{2}(1,1) \oplus \frac{1}{2}(0,0)$ and $q \equiv \frac{1}{2}(1,0) \oplus \frac{1}{2}(0,1)$ in Figure 2. while the EIA model predicts that $p$ is strictly preferred to $q$ if and only if $\delta<1$ and $(\alpha, \beta) \neq \mathbf{0}$.

Borah (2011) proposes a model of social preferences under risk, in which a function $g$ describes a preference for equality of opportunity. The function $g$ is defined solely on the marginal distribution $p_{2}$ of a lottery $p$ on the other agent's allocations. Because of this feature, Borah's (2011) model implies unnatural preferences for equality of opportunity. ${ }^{12}$ For instance, two lotteries $q \equiv \frac{1}{2}(1,0) \oplus \frac{1}{2}(0,1)$ and $l \equiv \frac{1}{2}(1,0) \oplus \frac{1}{2}(1,1)$ must be equally desirable in terms of equality of opportunity, because $q_{2}=l_{2}$. (Both marginal distributions on the other agent's allocations are the same 50-50 lottery between zero and one.) However, it would be natural to say that the lottery $q$ is equal in opportunity but the lottery $l$ is unequal in opportunity,

\footnotetext{
${ }^{11}$ For $\alpha=1$ and $\beta=0.6$, which are assumed for 30 percent of the population in Fehr and Schmidt (1999), the condition is equivalent to $\delta \geq 0.875$. For $\alpha=2$ and $\beta=0.6$, which are assumed for 10 percent of the population in Fehr and Schmidt (1999), the condition is equivalent to $\delta \geq 0.93$.

${ }^{12}$ In a two-agent environment, Borah's (2011) model is $W(p)=\left(\sum_{\mathbf{x} \in \mathbb{R}^{I}} p(\mathbf{x}) w(\mathbf{x})\right)+g\left(p_{2}\right)$, where $w(\mathbf{x})$ captures a preference for equality of outcome, and $g\left(p_{2}\right)$ captures a preference for equality of opportunity. He calls $\sum_{\mathbf{x} \in \mathbb{R}^{I}} p(\mathbf{x}) w(\mathbf{x})$ baseline expected utility evaluation and $g\left(p_{2}\right)$ adjustment. He says "Such an adjustment may be needed because the expected utility evaluation only incorporates the decision maker's concern for end-outcomes and not opportunities."
} 
because in $q$ both agents have an equal chance to get one, but in $l$ only the decision maker gets one with certainty.

In contrast, the EIA model captures a preference for equality of opportunity through the term $U\left(E_{p_{1}}\left(x_{1}\right), E_{p_{2}}\left(x_{2}\right)\right)$. (Note that $E_{p}\left(x_{i}\right)=E_{p_{i}}\left(x_{i}\right)$ for each $i \in I$.) This term depends not only on $p_{2}$ but also on the marginal distribution $p_{1}$ of a lottery $p$ on the decision maker's allocations. Because of this difference between the EIA model and Borah's (2011) model, neither model is a special case of the other. In fact, if $\delta>0$ and $(\alpha, \beta) \neq \mathbf{0}$, then the EIA model predicts that the decision maker will feel disutilities from inequality in opportunity in the lottery $l \equiv \frac{1}{2}(1,0) \oplus \frac{1}{2}(1,1)$ but not in the lottery $q \equiv \frac{1}{2}(1,0) \oplus \frac{1}{2}(0,1)$.

In a deterministic environment, Rohde (2010) also obtains a characterization of Fehr and Schmidt's (1999) model. Rohde's (2010) axioms differ from ours. ${ }^{13}$ Rohde's (2010) results and ours were obtained simultaneously and independently. Moreover, since Rohde (2010) studies a deterministic environment, she does not consider the trade-off between the two notions of equality, which is the main issue in our article.

\section{Concluding Remark}

One important feature of the EIA model is that the model reduces into the function $U$ proposed by Fehr and Schmidt (1999) on the set of deterministic allocations. To conclude the article, we discuss an extension to incorporate other models of social preferences.

To state the extension, first we need to define a property of a function $U$ on $\mathbb{R}^{n}$. $U$ is constant linear, if $U(\lambda \mathbf{x}+(1-\lambda)(c, \ldots, c))=\lambda U(\mathbf{x})+(1-\lambda) U(c, \ldots, c)$ for all $\mathbf{x} \in \mathbb{R}^{n}, c \in \mathbb{R}$, and $\lambda \in[0,1]$. In Lemma 3 in the Appendix, we prove the following result. For any binary relation $\succsim$ on $\Delta\left(\mathbb{R}^{n}\right)$, if (i) there exists a concave and constant linear function $U$ on $\mathbb{R}^{n}$ that represents $\succsim$ on $\mathbb{R}^{n}$ (i.e., $\mathbf{x} \succsim \mathbf{y} \Leftrightarrow U(\mathbf{x}) \geq U(\mathbf{y})$ ) and (ii) $\succsim$ satisfies Rationality, Constant Independence for Probability Mixture, and Dominance, then there exists $\delta \in[0,1]$ such that $\succsim$ is represented by $V(p)$ $\equiv \delta U\left(E_{p}(\mathbf{x})\right)+(1-\delta) E_{p}(U(\mathbf{x}))$.

Note that Fehr and Schmidt's (1999) model satisfies the concavity and the certainty linearity. Charness and Rabin's (2002) model, $U^{C R}(\mathbf{x}) \equiv(1-\lambda) x_{1}+$ $\lambda\left(\gamma \sum_{i \in I} x_{i}+(1-\gamma) \min _{i \in I} x_{i}\right)$, also satisfies the two properties. Therefore, if the function $U^{C R}$ represents $\succsim$ on $\mathbb{R}^{n}$ and $\succsim$ satisfies the all properties in the condition (ii) above, then Lemma 3 shows that there exists $\delta \in[0,1]$ such that $\succsim$ is represented by $V(p) \equiv \delta U^{C R}\left(E_{p}(\mathbf{x})\right)+(1-\delta) E_{p}\left(U^{C R}(\mathbf{x})\right)$.

\section{ApPendix: Proof of Theorem ANd Remark}

To prove the main theorem and the remark, we will prove three lemmas. Lemma 1 provides the representation theorem of the model proposed by Fehr and Schmidt (1999). Lemma 2 shows a representation theorem on a subset of $\mathbb{R}^{2}$. By using

\footnotetext{
${ }^{13}$ Rohde (2010) assumes one axiom, Constant Equivalence, which we do not assume.
} 
Lemma 2, Lemma 3 proves the result mentioned in Section V. The proofs of the theorem and the remark are in Section D.

\section{A. Lemma 1}

LEMMA 1: A preference $\succsim$ on $\mathbb{R}^{n}$ satisfies Rationality, Inequality Aversion, and Quasi-Comonotonic Independence for Outcome Mixture if and only if there exist $(\alpha, \beta) \in \mathbb{R}_{+}^{n-1} \times \mathbb{R}_{+}^{n-1}$ such that $\succsim$ is represented by $U(\mathbf{x})=x_{1}-\sum_{i=2}^{n}\left(\alpha_{i} \max \left\{x_{i}-\right.\right.$ $\left.\left.x_{1}, 0\right\}+\beta_{i} \max \left\{x_{1}-x_{i}, 0\right\}\right)$.

\section{PROOF OF LEMMA 1:}

The necessity of the axioms is trivial. In the following, we show the sufficiency. Fix $\succsim$ that satisfies the axioms.

Step 1: There exists a utility function $U: \mathbb{R}^{n} \rightarrow \mathbb{R}$ such that (i) for all $\lambda \in[0,1]$ and $\mathbf{x}, \mathbf{y} \in \mathbb{R}^{n}$, if $\mathbf{x}$ and $\mathbf{y}$ are quasi-comonotonic, then $U(\lambda \mathbf{x}+(1-\lambda) \mathbf{y})$ $=\lambda U(\mathbf{x})+(1-\lambda) U(\mathbf{y})$, (ii) for all $\mathbf{x} \in \mathbb{R}^{n}$ and $a \in \mathbb{R}_{+}, U(a \mathbf{x})=a U(\mathbf{x})$, and (iii) $U$ is unique up to positive affine transformation.

\section{PROOF OF STEP 1:}

Since an equal allocation $(z, \ldots, z)$ is quasi-comonotonic with any other allocations, Quasi-Comonotonic Independence for Outcome Mixture implies that for all $\mathbf{x}, \mathbf{y} \in \mathbb{R}^{n}, z \in \mathbb{R}$, and $\lambda \in(0,1), \mathbf{x} \succsim \mathbf{y} \Leftrightarrow \lambda \mathbf{x}+(1-\lambda)(z, \ldots, z) \succsim \lambda \mathbf{y}+(1-\lambda)$ $\times(z, \ldots, z)$. By the standard argument with the von Neumann-Morgenstern Theorem, the above property implies that there exists a utility function $U$ on $\mathbb{R}^{n}$ such that for all $\mathbf{x} \in \mathbb{R}^{n}, z \in \mathbb{R}$, and $\lambda \in(0,1)$,

$$
U(\lambda \mathbf{x}+(1-\lambda)(z, \ldots, z))=\lambda U(\mathbf{x})+(1-\lambda) U(z, \ldots, z) .
$$

In addition, $U$ is unique up to positive affine transformation, so that (iii) holds. Hence, we can normalize $U$ by $U(1, \ldots, 1)=1$ and $U(-1, \ldots,-1)=-1$, without loss of generality. Then, $U(0, \ldots, 0)=0$. Therefore, (A1) shows that for all $\mathbf{x} \in \mathbb{R}$ and $a \in[0,1]$ such that $a \leq 1, U(a \mathbf{x})=a U(\mathbf{x})$. For all $x \in \mathbb{R}$ and $a>1$, $\mathbf{x} \sim \frac{1}{a}(a \mathbf{x})+\left(1-\frac{1}{a}\right)(0, \ldots, 0)$. Hence, $U(\mathbf{x})=\frac{1}{a} U(a \mathbf{x})$, so that $U(a \mathbf{x})=a U(\mathbf{x})$. Hence, (ii) holds.

To show (i), choose $\lambda \in[0,1]$ and $\mathbf{x}, \mathbf{y} \in \mathbb{R}^{n}$ such that $\mathbf{x}$ and $\mathbf{y}$ are quasicomonotonic. By (ii), there exists a positive integer $n$ such that $\frac{1}{n} U(\mathbf{x})$ $=U\left(\frac{1}{n} \mathbf{x}\right) \in[-1,1]$. Since $[-1,1] \subset\{U(z, \ldots, z) \mid z \in \mathbb{R}\}$, there exists $z \in \mathbb{R}$ such that $(z, \ldots, z) \sim \frac{1}{n} \mathbf{x}$. In addition, $\frac{1}{n} \mathbf{x}$ and $\frac{1}{n} \mathbf{y}$ are also quasi-comonotonic. Hence, Quasi-Comonotonic Independence for Outcome Mixture shows that $\lambda\left(\frac{1}{n} \mathbf{x}\right)+$ $(1-\lambda)\left(\frac{1}{n} \mathbf{y}\right) \sim \lambda(z, \ldots, z)+(1-\lambda)\left(\frac{1}{n} \mathbf{y}\right)$. Therefore, by using (A1) and (ii), we obtain $\frac{1}{n} U(\lambda \mathbf{x}+(1-\lambda) \mathbf{y})=U\left(\lambda\left(\frac{1}{n} \mathbf{x}\right)+(1-\lambda)\left(\frac{1}{n} \mathbf{y}\right)\right)=U(\lambda(z, \ldots, z)+$ $\left.(1-\lambda)\left(\frac{1}{n} \mathbf{y}\right)\right)=\lambda U(z, \ldots, z)+(1-\lambda) U\left(\frac{1}{n} \mathbf{y}\right)=\lambda\left(\frac{1}{n} U(\mathbf{x})\right)+(1-\lambda)\left(\frac{1}{n} U(\mathbf{y})\right)$, so that (i) holds. 
For all $i \neq 1$, define $\alpha_{i}=-U\left(1,(0)_{-i}\right)$ and $\beta_{i}=-U\left(-1,(0)_{-i}\right)$. By Inequality Aversion, $\alpha_{i} \geq 0$ and $\beta_{i} \geq 0$ for all $i \neq 1$. Fix any $\mathbf{x} \in \mathbb{R}^{n}$.

Step 2: Let $\bar{I}=\left\{i \in I \mid x_{i}>x_{1}\right\}$ and $\underline{I}=\left\{i \in I \mid x_{1}>x_{i}\right\}$. Then, (i) for all $i \in \bar{I}$, $U\left(x_{i}-x_{1},(0)_{-i}\right)=-\alpha_{i} \max \left\{x_{i}-x_{1}, 0\right\}$, (ii) for all $i \in \underline{I}, U\left(x_{i}-x_{1},(0)_{-i}\right)$ $=-\beta_{i} \max \left\{x_{1}-x_{i}, 0\right\}$, and (iii) for all $i \in I \backslash(\bar{I} \cup \underline{I}), U\left(x_{i}-x_{1},(0)_{-i}\right)=0$.

\section{PROOF OF STEP 2:}

To show (i), fix $i \in \bar{I}$. By Step 1 (ii), $U\left(x_{i}-x_{1},(0)_{-i}\right)=\left(x_{i}-x_{1}\right) U\left(1,(0)_{-i}\right)$ $=-\alpha_{i} \max \left\{x_{i}-x_{1}, 0\right\}$. (ii) and (iii) can be proved in the same way.

By the steps, the following calculations complete the proof of Lemma 1. Note that $\frac{1}{n} \mathbf{x} \sim \frac{1}{n}\left(x_{1}, \ldots, x_{1}\right)+\sum_{i=2}^{n} \frac{1}{n}\left(x_{i}-x_{1},(0)_{-i}\right)$ and any pair among $\left\{\left(x_{1}, \ldots, x_{1}\right),\left(x_{2}-x_{1},(0)_{-2}\right), \ldots,\left(x_{n}-x_{1},(0)_{-n}\right)\right\}$ is quasi-comonotonic. Therefore, $U(\mathbf{x})=n U\left(\frac{1}{n} \mathbf{x}\right)=n U\left(\frac{1}{n}\left(x_{1}, \ldots, x_{1}\right)+\sum_{i=2}^{n} \frac{1}{n}\left(x_{i}-x_{1},(0)_{-i}\right)\right)=U\left(x_{1}, \ldots, x_{1}\right)+$ $\sum_{i=2}^{n} U\left(x_{i}-x_{1},(0)_{-i}\right)=x_{1}-\sum_{i=2}^{n}\left(\alpha_{i} \max \left\{x_{i}-x_{1}, 0\right\}+\beta_{i} \max \left\{x_{1}-x_{i}, 0\right\}\right)$.

\section{B. Lemma 2}

Let $\mathscr{D}$ be a subset of $\mathbb{R}^{2}$ and let $\hat{\succsim}$ be a binary relation on $\mathscr{D} . \hat{\succ}$ and $\hat{\sim}$ denote the asymmetric and symmetric parts of $\hat{\succsim}$, respectively.

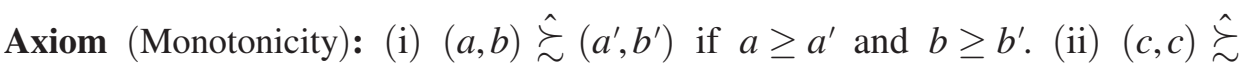
$\left(c^{\prime}, c^{\prime}\right)$ if and only if $c \geq c^{\prime}$.

Axiom (Constant Independence): For all $(a, b),\left(a^{\prime}, b^{\prime}\right),(c, c) \in \mathscr{D}$ and $\lambda \in[0,1]$, $(a, b) \hat{\succsim}\left(a^{\prime}, b^{\prime}\right)$ if and only if $\lambda(a, b)+(1-\lambda)(c, c) \hat{\succsim} \lambda\left(a^{\prime}, b^{\prime}\right)+(1-\lambda)(c, c)$.

Axiom (Constant Continuity): For all $(a, b),\left(a^{\prime}, b^{\prime}\right),(c, c) \in \mathscr{D}$, if $(a, b) \hat{\succsim}$ $\left(a^{\prime}, b^{\prime}\right) \hat{\succsim}(c, c)$ or $(c, c) \hat{\succsim}\left(a^{\prime}, b^{\prime}\right) \hat{\succsim}(a, b)$, then there exists $\lambda \in[0,1]$ such that $\left(a^{\prime}, b^{\prime}\right) \hat{\sim} \lambda(a, b)+(1-\lambda)(c, c)$.

LEMMA 2: Suppose that $(i)$ for any $(a, b) \in \mathscr{D}, a \geq b,($ ii $)$ for all $(a, b),(c, c) \in \mathscr{D}$ and $\lambda \in[0,1], \lambda(a, b)+(1-\lambda)(c, c) \in \mathscr{D}$, and $(i i i)(c, c) \in \mathscr{D}$ for any $c \in \mathbb{R}$. If a preference $\hat{\succsim}$ on $\mathscr{D}$ satisfies Completeness, Transitivity, Monotonicity, Constant Independence, and Constant Continuity, then there exists $\delta \in[0,1]$ such that $(a, b) \hat{\succsim}\left(a^{\prime}, b^{\prime}\right) \Leftrightarrow \delta a+(1-\delta) b \geq \delta a^{\prime}+(1-\delta) b^{\prime}$.

\section{PROOF OF LEMMA 2:}

If $(a, a) \hat{\sim}(a, b)$ for any $(a, b) \in \mathscr{D}$, then by Monotonicity $(\mathrm{i}),(a, b) \hat{\succsim}\left(a^{\prime}, b^{\prime}\right)$ $\Leftrightarrow(a, a) \hat{\succsim}\left(a^{\prime}, a^{\prime}\right) \Leftrightarrow a \geq a^{\prime}$. Hence, Lemma 2 holds for $\delta=1$.

In the following, we assume $\left(a^{*}, a^{*}\right) \hat{\succ}\left(a^{*}, b^{*}\right)$ for some $\left(a^{*}, b^{*}\right) \in \mathscr{D}$. (Note that by assumption (iii), $\left(a^{*}, a^{*}\right) \in \mathscr{D}$.) This assumption implies that $a^{*} \neq b^{*}$. Then, by assumption (i), $a^{*}>b^{*}$. By assumption (iii), $\bar{c}>a^{*}$ for some $\bar{c}$. Monotonicity (ii) 


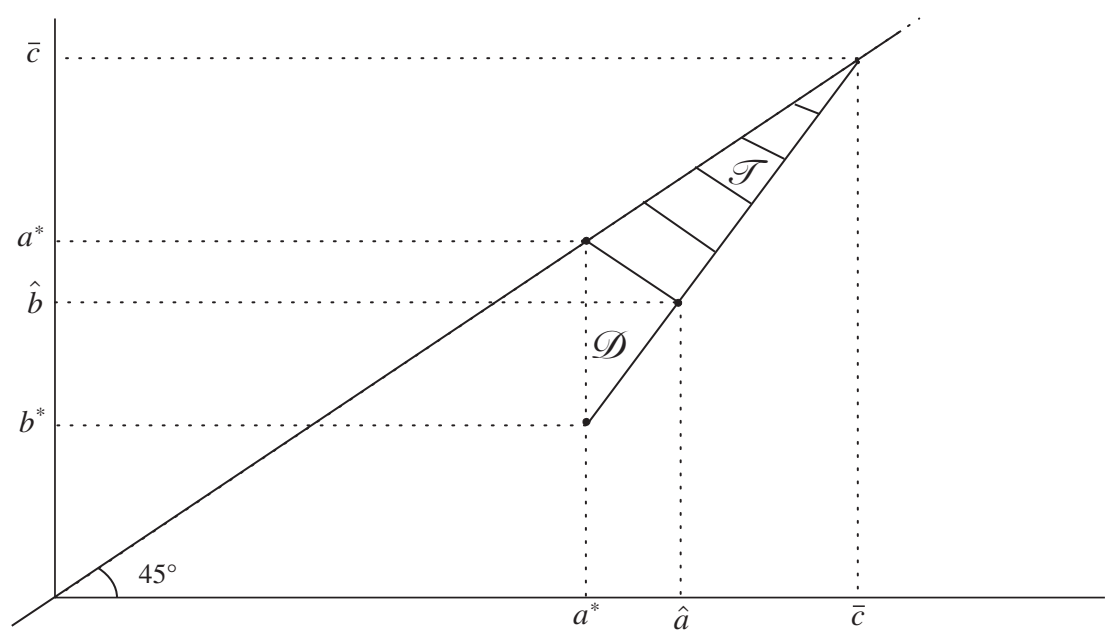

Figure A1. IndifFerence Curves of $\hat{\succsim}$ on $\mathscr{T}$

shows $(\bar{c}, \bar{c}) \hat{\succ}\left(a^{*}, a^{*}\right)$. Then by Constant Continuity, there exist $\bar{\lambda} \in(0,1)$ such that $\left(a^{*}, a^{*}\right) \hat{\sim} \bar{\lambda}\left(a^{*}, b^{*}\right)+(1-\bar{\lambda})(\bar{c}, \bar{c})$. Define $\hat{a}=\bar{\lambda} a^{*}+(1-\bar{\lambda}) \bar{c}$ and $\hat{b}=\bar{\lambda} b^{*}+$ $(1-\bar{\lambda}) \bar{c}$. Then, $(\hat{a}, \hat{b}) \hat{\sim}\left(a^{*}, a^{*}\right), \hat{a}>a^{*}$, and $\hat{a}>\hat{b}$.

Let $\mathscr{T}$ be a triangle including the interior which consists of the vertices $(\bar{c}, \bar{c})$, $\left(a^{*}, a^{*}\right)$, and $(\hat{a}, \hat{b})$. (Formally, $\mathscr{T}=\left\{(a, b) \in \mathbb{R}^{2} \mid a \geq b,\left\langle\left(a^{*}-\hat{a}, \hat{b}-a^{*}\right),(a, b)-\right.\right.$ $\left.\left.\left(a^{*}, a^{*}\right)\right\rangle \geq 0,\langle(\bar{c}-\hat{a}, \hat{b}-\bar{c}),(a, b)-(\bar{c}, \bar{c})\rangle \geq 0\right\}$, where $\langle\cdot, \cdot\rangle$ is an inner product.) In the following, we show the existence of the desired $\delta \in[0,1]$ on $\mathscr{T}$ and then extend the result on $\mathscr{D}$.

Step 1: (i) $\left(a^{*}, a^{*}\right) \neq(\hat{a}, \hat{b}) \neq(\bar{c}, \bar{c})$; (ii) if $(a, b) \in \mathscr{T}$, then $(a, b) \in \mathscr{D}$.

\section{PROOF OF STEP 1:}

Since $a^{*}>b^{*}$ and $\bar{\lambda}>0, \hat{a}>\hat{b}$. Therefore, $\left(a^{*}, a^{*}\right) \neq(\hat{a}, \hat{b}) \neq(\bar{c}, \bar{c})$. Let $(a, b) \in \mathscr{T}$ to show $(a, b) \in \mathscr{D}$. Since $\mathscr{T}$ is a triangle, there exist $\lambda, \mu \in[0,1]$ such that $(a, b)$ $=\lambda(\bar{c}, \bar{c})+\mu\left(a^{*}, a^{*}\right)+(1-\lambda-\mu)(\hat{a}, \hat{b})=(\lambda+\mu)(c, c)+(1-\lambda-\mu)(\hat{a}, \hat{b})$, where $c \equiv \frac{\lambda}{\lambda+\mu} \bar{c}+\frac{\mu}{\lambda+\mu} a^{*}$. Since $(\hat{a}, \hat{b}) \in \mathscr{D}$, it follows from assumptions (ii) and (iii) that $(a, b) \in \mathscr{D}$.

Step 2: There exists $\delta \in[0,1]$ such that for any $(a, b),\left(a^{\prime}, b^{\prime}\right) \in \mathscr{T},(a, b) \hat{\succsim}$ $\left(a^{\prime}, b^{\prime}\right) \Leftrightarrow \delta a+(1-\delta) b \geq \delta a^{\prime}+(1-\delta) b^{\prime}$.

\section{PROOF OF STEP 2:}

Substep 2.1: For all $(a, b) \in \mathscr{T}$, there exists a unique number $\lambda \in[0,1]$ such that $(a, b) \hat{\sim} \lambda(\bar{c}, \bar{c})+(1-\lambda)\left(a^{*}, a^{*}\right)$.

\section{PROOF OF SUBSTEP 2.1:}

Choose any $(a, b) \in \mathscr{T}$. Since $\mathscr{T}$ is the triangle, there exist $\lambda, \mu \in[0,1]$ such that $(a, b)=\lambda(\bar{c}, \bar{c})+\mu\left(a^{*}, a^{*}\right)+(1-\lambda-\mu)(\hat{a}, \hat{b})$. Since $(\hat{a}, \hat{b}) \hat{\sim}\left(a^{*}, a^{*}\right)$, Transitivity 
and Constant Independence show $(a, b) \hat{\sim} \lambda(\bar{c}, \bar{c})+(1-\lambda)\left(a^{*}, a^{*}\right)$. Since $\bar{c}>a^{*}$, Monotonicity (ii) shows that $\lambda$ is unique. Hence, Substep 2.1 is proved.

For all $(a, b) \in \mathscr{T}$, define $c(a, b)=\lambda \bar{c}+(1-\lambda) a^{*}$, where $\lambda$ is obtained in Substep 2.1.

Substep 2.2: For all $(a, b) \in \mathscr{T}, \frac{a-c(a, b)}{a-b}=\frac{\hat{a}-a^{*}}{\hat{a}-\hat{b}}$.

\section{PROOF OF SUBSTEP 2.2:}

Choose any $(a, b) \in \mathscr{T}$. Since $c(a, b)=\lambda \bar{c}+(1-\lambda) a^{*}$, $\frac{a-c(a, b)}{a-b}=\frac{a-\lambda \bar{c}-(1-\lambda) a^{*}}{a-b}$. Since $(a, b) \in \mathscr{T},(a, b)=\lambda^{\prime}(\bar{c}, \bar{c})+\mu\left(a^{*}, a^{*}\right)+$ $\left(1-\lambda^{\prime}-\mu\right)(\hat{a}, \hat{b})$ for some $\lambda^{\prime}, \mu \in[0,1]$. Since $\left(a^{*}, a^{*}\right) \hat{\sim}(\hat{a}, \hat{b})$, Transitivity and Constant Independence show $(a, b) \hat{\sim} \lambda^{\prime}(\bar{c}, \bar{c})+\left(1-\lambda^{\prime}\right)\left(a^{*}, a^{*}\right)$. Hence, Substep 2.1 shows $\lambda^{\prime}=\lambda$, so that $(a, b)=\lambda(\bar{c}, \bar{c})+\mu\left(a^{*}, a^{*}\right)+(1-\lambda-\mu)(\hat{a}, \hat{b})$. Thus, $a-\lambda \bar{c}-(1-\lambda) a^{*}=\left(\lambda \bar{c}+\mu a^{*}+(1-\lambda-\mu) \hat{a}\right)-\lambda \bar{c}-(1-\lambda) a^{*}$ $=(1-\lambda-\mu)\left(\hat{a}-a^{*}\right)$ and $a-b=(1-\lambda-\mu)(\hat{a}-\hat{b})$. Hence, $\frac{a-\lambda \bar{c}-(1-\lambda) a^{*}}{a-b}=\frac{\hat{a}-a^{*}}{\hat{a}-\hat{b}}$, so that Substep 2.2 is proved.

Define

$$
\delta=\frac{a^{*}-\hat{b}}{\hat{a}-\hat{b}} .
$$

Substep 2.3: $\delta \in[0,1]$.

\section{PROOF OF SUBSTEP 2.3:}

Since $\hat{a}>\hat{b}$ and $\hat{a} \geq a^{*}$ by definitions, $\delta=1-\frac{\hat{a}-a^{*}}{\hat{a}-\hat{b}} \leq 1$. To show $\delta \geq 0$, it suffices to prove $a^{*} \geq \hat{b}$. Suppose by way of contradiction that $a^{*}<\hat{b}$. Remember that $a^{*}<\hat{a}$ and $\left(a^{*}, a^{*}\right) \hat{\sim}(\hat{a}, \hat{b})$. There exists a positive $\varepsilon$ such that $\min \left\{\hat{a}-a^{*}, \hat{b}-a^{*}\right\}>$ $\varepsilon$. Then, $\hat{a}>a^{*}+\varepsilon$ and $\hat{b}>a^{*}+\varepsilon$. By Monotonicity (i), $(\hat{a}, \hat{b}) \hat{\succsim}\left(a^{*}+\varepsilon, a^{*}+\varepsilon\right)$. However, by Monotonicity (ii), $\left(a^{*}+\varepsilon, a^{*}+\varepsilon\right) \hat{\succ}\left(a^{*}, a^{*}\right) \hat{\sim}(\hat{a}, \hat{b}) \hat{\succsim}\left(a^{*}+\varepsilon, a^{*}+\varepsilon\right)$, which is a contradiction. Hence, $a^{*} \geq \hat{b}$. Then, $\delta \in[0,1]$.

Substep 2.4: For any $(a, b),\left(a^{\prime}, b^{\prime}\right) \in \mathscr{T},(a, b) \hat{\succsim}\left(a^{\prime}, b^{\prime}\right) \Leftrightarrow \delta a+(1-\delta) b \geq$ $\delta a^{\prime}+(1-\delta) b^{\prime}$.

\section{PROOF OF SUBSTEP 2.4:}

By substituting $\frac{\hat{a}-a^{*}}{\hat{a}-\hat{b}}=1-\delta$ into the result of Substep 2.2, we conclude that for all $(a, b) \in \mathscr{T}, c(a, b)=\delta a+(1-\delta) b$. Therefore, for any $(a, b),\left(a^{\prime}, b^{\prime}\right) \in \mathscr{T}$ by Substeps 2.1 and 2.2, $(a, b) \hat{\succsim}\left(a^{\prime}, b^{\prime}\right) \Leftrightarrow c(a, b) \geq c\left(a^{\prime}, b^{\prime}\right) \Leftrightarrow \delta a+(1-\delta) b \geq$ $\delta a^{\prime}+(1-\delta) b^{\prime}$.

Substeps 2.3 and 2.4 establish Step 2.

Step 3: For all $(a, b),\left(a^{\prime}, b^{\prime}\right) \in \mathscr{D},(a, b) \hat{\succsim}\left(a^{\prime}, b^{\prime}\right) \Leftrightarrow \delta a+(1-\delta) b \geq \delta a^{\prime}+$ $(1-\delta) b^{\prime}$. 


\section{PROOF OF STEP 3:}

Let $c^{*}=\frac{1}{2} \bar{c}+\frac{1}{2} a^{*}$. Choose any $(a, b),\left(a^{\prime}, b^{\prime}\right) \in \mathscr{D}$. By assumption (i), $a \geq b$ and $a^{\prime} \geq b^{\prime}$. Hence, there exists $\lambda \in(0,1]$ such that $\lambda(a, b)+(1-\lambda)\left(c^{*}, c^{*}\right)$ and $\lambda\left(a^{\prime}, b^{\prime}\right)+(1-\lambda)\left(c^{*}, c^{*}\right)$ belong to $\mathscr{T}$. Step 2 and Constant Independence show $(a, b) \hat{\succsim}\left(a^{\prime}, b^{\prime}\right) \Leftrightarrow \lambda(a, b)+(1-\lambda)\left(c^{*}, c^{*}\right) \hat{\succsim} \lambda\left(a^{\prime}, b^{\prime}\right)+(1-\lambda)\left(c^{*}, c^{*}\right) \Leftrightarrow$ $\delta\left(\lambda a+(1-\lambda) c^{*}\right)+(1-\delta)\left(\lambda b+(1-\lambda) c^{*}\right) \geq \delta\left(\lambda a^{\prime}+(1-\lambda) c^{*}\right)+(1-\delta)$ $\times\left(\lambda b^{\prime}+(1-\lambda) c^{*}\right) \Leftrightarrow \delta a+(1-\delta) b \geq \delta a^{\prime}+(1-\delta) b^{\prime}$.

\section{Lemma 3}

Let $\succsim$ be a binary relation on $\Delta\left(\mathbb{R}^{n}\right)$.

LEMMA 3: Suppose that $(i)$ there exists a concave and constant linear function $U$ on $\mathbb{R}^{n}$ that represents $\succsim$ on $\mathbb{R}^{n}$ (i.e., $\left.\mathbf{x} \succsim \mathbf{y} \Leftrightarrow U(\mathbf{x}) \geq U(\mathbf{y})\right)$ and $(i i) \succsim$ satisfies Rationality, Constant Independence for Probability Mixture, and Dominance. Then, there exists $\delta \in[0,1]$ such that $\succsim$ is represented by $\delta U\left(E_{p}(\mathbf{x})\right)+(1-\delta) E_{p}(U(\mathbf{x}))$.

\section{PROOF OF LEMMA 3:}

Step 1: There exists a utility function $V$ such that $(\mathrm{i}) V(\lambda p \oplus(1-\lambda)(x, \ldots, x))$ $=\lambda V(p)+(1-\lambda) V(x, \ldots, x)$ for all $p \in \Delta\left(\mathbb{R}^{n}\right), x \in \mathbb{R}$, and $\lambda \in[0,1]$; (ii) $V=U$ on $\mathbb{R}^{n}$.

\section{PROOF OF STEP 1:}

As in Step 1 of the proof of Lemma 1, Constant Independence for Probability Mixture implies that there exists a utility function $V$ such that for all $p \in \Delta\left(\mathbb{R}^{n}\right)$, $x \in \mathbb{R}$, and $\lambda \in[0,1], V(\lambda p \oplus(1-\lambda)(x, \ldots, x))=\lambda V(p)+(1-\lambda) V(x, \ldots, x)$, so that (i) holds. In addition, $V$ is unique up to positive affine transformation. Hence, we can normalize $V$ by $V(1, \ldots, 1)=1$ and $V(-1, \ldots,-1)=-1$, without loss of generality.

To prove (ii), it suffices to show that $V=U$ on $\{(x, \ldots, x) \mid x \in \mathbb{R}\}$ because $V$ is unique up to positive affine transformation. Remember $U(\lambda \mathbf{y}+(1-\lambda)(x, \ldots, x))$ $=\lambda U(\mathbf{y})+(1-\lambda) x$ for all $\mathbf{y} \in \mathbb{R}^{n}, x \in \mathbb{R}$, and $\lambda \in[0,1]$. Hence, $\lambda=0$ implies $U(x, \ldots, x)=x$. Therefore, it suffices to show $V(x, \ldots, x)=x$ for all $x \in \mathbb{R}$.

Note that for all $x, y \in \mathbb{R}, V(x, \ldots, x) \geq V(y, \ldots, y) \Leftrightarrow(x, \ldots, x) \succsim(y, \ldots, y) \Leftrightarrow$ $x \geq y$. Hence, there exists an increasing function $f: \mathbb{R} \rightarrow \mathbb{R}$ such that $V(x, \ldots, x)$ $=f(x)$ for all $x \in \mathbb{R}$. To show that $f$ is the identity function, note that Dominance trivially implies $\lambda(x, \ldots, x)+(1-\lambda)(y, \ldots, y) \sim \lambda(x, \ldots, x) \oplus(1-\lambda)(y, \ldots, y)$ for all $x, y \in \mathbb{R}$ and $\lambda \in[0,1]$. Therefore, by property (i) of $V$, for any $x, y \in \mathbb{R}$ and $\lambda \in[0,1], \lambda f(x)+(1-\lambda) f(y)=\lambda V(x, \ldots, x)+(1-\lambda) V(y, \ldots, y)=V(\lambda(x, \ldots, x) \oplus$ $(1-\lambda)(y, \ldots, y))=V(\lambda(x, \ldots, x)+(1-\lambda)(y, \ldots, y))=f(\lambda x+(1-\lambda) y)$. Hence, $f$ is affine. In addition, by the normalizations, $f(1)=1$ and $f(-1)=-1$. Therefore, $f$ is the identity function, or $V(x, \ldots, x)=x$ for all $x \in \mathbb{R}$.

To use Lemma 2, define

$$
\mathscr{D}=\left\{\left(U\left(E_{p}(\mathbf{x})\right), E_{p}(U(\mathbf{x}))\right) \in \mathbb{R}^{2} \mid p \in \Delta\left(\mathbb{R}^{n}\right)\right\}
$$


Step 2: $\mathscr{D}$ satisfies the properties (i), (ii), and (iii) in Lemma 2.

\section{PROOF OF STEP 2:}

To show (i), it suffices to show that $U\left(E_{p}(\mathbf{x})\right) \geq E_{p}(U(x))$ for all $p \in \Delta\left(\mathbb{R}^{n}\right)$. Since $U$ is concave, Jensen's Inequality shows that $U\left(E_{p}(\mathbf{x})\right) \geq E_{p}(U(\mathbf{x}))$ for all $p \in \Delta\left(\mathbb{R}^{n}\right)$. Property (ii) holds by the constant linearity of $U$. To see (iii) holds, note that for any $x \in \mathbb{R}$, if $p \equiv \delta_{(x, \ldots, x)}$, then $U\left(E_{p}(\mathbf{x})\right)=x=E_{p}(U(\mathbf{x}))$.

To define a binary relation $\hat{\succsim}$ on $\mathscr{D}$, we define a function $v$ on $\mathscr{D}$. For all $(a, b) \in \mathscr{D}$, define

$$
v(a, b)=V(p)
$$

where $p \in \Delta\left(\mathbb{R}^{n}\right)$ such that $U\left(E_{p}(\mathbf{x})\right)=a$ and $E_{p}(U(\mathbf{x}))=b$.

Step 3: $v$ is a well-defined weakly increasing function.

\section{PROOF OF STEP 3:}

To show that $v$ is well defined, it suffices to show that $\left(U\left(E_{p}(\mathbf{x})\right), E_{p}(U(\mathbf{x}))\right)$ $=\left(U\left(E_{q}(\mathbf{x})\right), E_{q}(U(\mathbf{x}))\right)$ implies $V(p)=V(q)$. Let $p, q \in \Delta\left(\mathbb{R}^{n}\right)$ such that $\left(U\left(E_{p}(\mathbf{x})\right)\right.$, $\left.E_{p}(U(\mathbf{x}))\right)=\left(U\left(E_{q}(\mathbf{x})\right), E_{q}(U(\mathbf{x}))\right)$. Then, both conditions (i) and (ii) in Dominance are satisfied with equalities. Therefore, Dominance shows $V(p)=V(q)$. Weak increasingness directly follows from Dominance.

Now, we define a binary relation $\hat{\succsim}$ on $\mathscr{D}$. For all $(a, b),\left(a^{\prime}, b^{\prime}\right) \in \mathscr{D}$ define

$$
(a, b) \hat{\grave{ }}\left(a^{\prime}, b^{\prime}\right) \Leftrightarrow v(a, b) \geq v\left(a^{\prime}, b^{\prime}\right) .
$$

Step 4: $\hat{\succsim}$ satisfies the all properties in Lemma 2 (i.e., Completeness, Transitivity, Monotonicity, Constant Independence, and Constant Continuity).

\section{PROOF OF STEP 4:}

Since $v$ is a well-defined increasing function, $\hat{\succsim}$ satisfies Completeness, Transitivity, and Monotonicity (i). Moreover, $(c, c) \hat{\succsim}\left(c^{\prime}, c^{\prime}\right) \Leftrightarrow v(c, c) \geq v\left(c^{\prime}, c^{\prime}\right) \Leftrightarrow$ $V(c, \ldots, c) \geq V\left(c^{\prime}, \ldots, c^{\prime}\right) \Leftrightarrow c \geq c^{\prime}$; hence, Monotonicity (ii) is also satisfied.

Constant Independence follows from Step 1 and the constant linearity of $U$. To see this, choose any $(a, b),\left(a^{\prime}, b^{\prime}\right),(z, z) \in \mathscr{D}$ and $\lambda \in[0,1]$. Then, there exist lotteries $p$ and $q$ such that $(a, b)=\left(U\left(E_{p}(\mathbf{x})\right), E_{p}(U(\mathbf{x}))\right)$ and $\left(a^{\prime}, b^{\prime}\right)=\left(U\left(E_{q}(\mathbf{x})\right)\right.$, $\left.E_{q}(U(\mathbf{x}))\right)$. To simplify notation, let $p^{\prime}=\lambda p \oplus(1-\lambda)(z, \ldots, z)$ and $q^{\prime}=\lambda q \oplus(1-\lambda)$ $\times(z, \ldots, z)$. By Step $1(\mathrm{i})$, then $(a, b) \succsim\left(a^{\prime}, b^{\prime}\right) \Leftrightarrow V(p) \geq V(q) \Leftrightarrow V\left(p^{\prime}\right) \geq V\left(q^{\prime}\right) \Leftrightarrow$ $\left(U\left(E_{p^{\prime}}(\mathbf{x})\right), E_{p^{\prime}}(U(\mathbf{x}))\right) \hat{\succsim}\left(U\left(E_{q^{\prime}}(\mathbf{x})\right), E_{q^{\prime}}(U(\mathbf{x}))\right)$. Moreover, by the constant linearity of $U,\left(U\left(E_{p^{\prime}}(\mathbf{x})\right), E_{p^{\prime}}(U(\mathbf{x}))\right)=\lambda(a, b)+(1-\lambda)(z, z)$ and $\left(U\left(E_{q^{\prime}}(\mathbf{x})\right), E_{q^{\prime}}(U(\mathbf{x}))\right)$ $=\lambda\left(a^{\prime}, b^{\prime}\right)+(1-\lambda)(z, z)$. This completes the proof of Constant Independence.

Finally, we show that $\succsim$ satisfies Constant Continuity. Choose any $(a, b),\left(a^{\prime}, b^{\prime}\right)$, $(z, z) \in \mathscr{D}$ such that $(\widetilde{a, b}) \hat{\succsim}\left(a^{\prime}, b^{\prime}\right) \hat{\succsim}(z, z)$. Then, there exist $p, q \in \Delta\left(\mathbb{R}^{n}\right)$ 
such that $\left(U\left(E_{p}(\mathbf{x})\right), E_{p}(U(\mathbf{x}))\right)=(a, b)$ and $\left(U\left(E_{q}(\mathbf{x})\right), E_{q}(U(\mathbf{x}))\right)=\left(a^{\prime}, b^{\prime}\right)$. Then $p \succsim q \succsim(z, \ldots, z)$. Then by Continuity, there exists $\lambda \in[0,1]$ such that $q \sim \lambda p \oplus$ $(1-\lambda)(z, \ldots, z)$. By definition, this implies that $\left(a^{\prime}, b^{\prime}\right) \hat{\sim} \lambda(a, b)+(1-\lambda)(z, z)$. The other case where $(z, z) \hat{\succsim}\left(a^{\prime}, b^{\prime}\right) \hat{\succsim}(a, b)$ is proved in the same way.

Given Steps 2 and 4 and Lemma 2, it follows that $p \succsim q \Leftrightarrow V(p) \geq V(q) \Leftrightarrow$ $\left(U\left(E_{p}(\mathbf{x})\right), E_{p}(U(\mathbf{x}))\right) \hat{\succsim}\left(U\left(E_{q}(\mathbf{x})\right), E_{q}(U(\mathbf{x}))\right) \Leftrightarrow \delta U\left(E_{p}(\mathbf{x})\right)+(1-\delta) E_{p}(U(\mathbf{x})) \geq$ $\delta U\left(E_{q}(\mathbf{x})\right)+(1-\delta) E_{q}(U(\mathbf{x}))$ for all $p, q \in \Delta\left(\mathbb{R}^{n}\right)$. This completes the proof of Lemma 3.

\section{Proof of Theorem and Remark}

The necessity of the axioms is trivial. To show Continuity, note that the EIA model is a weighted sum of max functions. The sufficiency follows from Lemmas 1 and 3 because $U(\mathbf{x})=x_{1}-\sum_{i=2}^{n}\left(\alpha_{i} \max \left\{x_{i}-x_{1}, 0\right\}+\beta_{i} \max \left\{x_{1}-x_{i}, 0\right\}\right)$ is concave and constant linear. ${ }^{14}$

In the following, we provide the proof of Remark. Suppose that $(\delta, \alpha, \beta)$ and $\left(\delta^{\prime}, \alpha^{\prime}, \beta^{\prime}\right)$ represent $\succsim$. Let $V$ and $V^{\prime}$ be the functions defined by $(\delta, \alpha, \beta)$ and $\left(\delta^{\prime}, \alpha^{\prime}, \beta^{\prime}\right)$, respectively.

First, we show $(\alpha, \beta)=\left(\alpha^{\prime}, \beta^{\prime}\right)$. Suppose to the contrary that $(\alpha, \beta) \neq\left(\alpha^{\prime}, \beta^{\prime}\right)$. Assume $\alpha_{i}^{\prime}>\alpha_{i}$ for some $i \neq 1$, without loss of generality. Therefore, $V\left(1,(0)_{-i}\right)$ $=-\alpha_{i}=V\left(-\alpha_{i}, \ldots,-\alpha_{i}\right)=-\alpha_{i}>-\alpha_{i}^{\prime}=V\left(-\alpha_{i}^{\prime}, \ldots,-\alpha_{i}^{\prime}\right)$ and $V^{\prime}\left(1,(0)_{-i}\right)=-\alpha_{i}^{\prime}$ $=V^{\prime}\left(-\alpha_{i}^{\prime}, \ldots,-\alpha_{i}^{\prime}\right)$. Thus, $\left(1,(0)_{-i}\right) \sim\left(-\alpha_{i}, \ldots,-\alpha_{i}\right) \succ\left(-\alpha_{i}^{\prime}, \ldots,-\alpha_{i}^{\prime}\right) \sim\left(1,(0)_{-i}\right)$, which is a contradiction.

Second, we show that if $(\alpha, \beta) \neq \mathbf{0}$, then $\delta=\delta^{\prime}$. Suppose to the contrary that $(\alpha, \beta) \neq 0$ but $\delta \neq \delta^{\prime}$. Without loss of generality, assume $\delta>\delta^{\prime}$ and $\alpha_{i}>0$ for some $i \neq 1$. Then, $V\left(\frac{1}{2}\left(1,(0)_{-i}\right) \oplus \frac{1}{2}\left(-1,(0)_{-i}\right)\right)=-\frac{1}{2}(1-\delta)\left(\alpha_{i}+\beta_{i}\right)$ $=V\left(-\frac{1}{2}(1-\delta)\left(\alpha_{i}+\beta_{i}\right), \ldots,-\frac{1}{2}(1-\delta)\left(\alpha_{i}+\beta_{i}\right)\right)$. Therefore, $\frac{1}{2}\left(1,(0)_{-i}\right) \oplus$ $\frac{1}{2}\left(-1,(0)_{-i}\right) \sim\left(-\frac{1}{2}(1-\delta)\left(\alpha_{i}+\beta_{i}\right), \ldots,-\frac{1}{2}(1-\delta)\left(\alpha_{i}+\beta_{i}\right)\right) \succ\left(-\frac{1}{2}\left(1-\delta^{\prime}\right)\right.$ $\left.\times\left(\alpha_{i}+\beta_{i}\right), \ldots,-\frac{1}{2}\left(1-\delta^{\prime}\right)\left(\alpha_{i}+\beta_{i}\right)\right) \sim \frac{1}{2}\left(1,(0)_{-i}\right) \oplus \frac{1}{2}\left(-1,(0)_{-i}\right)$, which is a contradiction.

\section{REFERENCES}

Bohnet, Iris, Fiona Greig, Benedikt Herrmann, and Richard Zeckhauser. 2008. "Betrayal Aversion: Evidence from Brazil, China, Oman, Switzerland, Turkey, and the United States." American Economic Review 98 (1): 294-310.

Bolton, Gary E., and Axel Ockenfels. 2010. "Betrayal Aversion: Evidence from Brazil, China, Oman, Switzerland, Turkey, and the United States: Comment." American Economic Review 100 (1): 628-33.

Borah, Abhinash. 2011. "Other-Regarding Preferences, Concerns for Others' Opportunities and Violations of Stochastic Dominance: A Choice-Theoretic Analysis.” Unpublished.

\footnotetext{
${ }^{14}$ To see the concavity, remember (i) for any convex function $\phi$ and positive number $a, a \max \{\phi(\cdot), 0\}$ is convex; (ii) a sum of convex functions is convex; (iii) for any convex function $\phi,-\phi$ is concave. By (i), $\alpha_{i} \max \left\{x_{i}-x_{1}, 0\right\}$ and $\beta_{i} \max \left\{x_{1}-x_{i}, 0\right\}$ are convex. Then, by (ii), $\alpha_{i} \max \left\{x_{i}-x_{1}, 0\right\}+\beta_{i} \max \left\{x_{1}-x_{i}, 0\right\}$ is convex. Since $U$ is a sum of concave functions, $U$ is also concave.
} 
Camerer, Colin F. 2003. Behavioral Game Theory: Experiments in Strategic Interaction. Princeton, NJ: Princeton University Press.

Charness, Gary, and Matthew Rabin. 2002. "Understanding Social Preferences with Simple Tests." Quarterly Journal of Economics 117 (3): 817-69.

Diamond, Peter A. 1967. "Cardinal Welfare, Individualistic Ethics, and Interpersonal Comparison of Utility: Comment.” Journal of Political Economy 75 (5): 765-66.

-Fehr, Ernst, Alexander Klein, and Klaus M. Schmidt. 2007. "Fairness and Contract Design." Econometrica 75 (1): 121-54.

Fehr, Ernst, and Klaus M. Schmidt. 1999. "A Theory of Fairness, Competition, and Cooperation." Quarterly Journal of Economics 114 (3): 817-68.

Fehr, Ernst, and Klaus M. Schmidt. 2006. "The Economics of Fairness, Reciprocity and Altruism -Experimental Evidence and New Theories.” In Handbook on the Economics of Giving, Altruism and Reciprocity, edited by Serge-Christophe Kolm and Jean Mercier Ythier, 615-91. Amsterdam: Elsevier, North-Holland.

Fudenberg, Drew, and David K. Levine. 2012. "Fairness, Risk Preferences and Independence: Impossibility Theorems." Journal of Economic Behavior and Organization 81 (2): 606-12.

Gajdos, Thibault, and Eric Maurin. 2004. "Unequal Uncertainties and Uncertain Inequalities: An Axiomatic Approach.” Journal of Economic Theory 116 (1): 93-118.

Grund, Christian, and Dirk Sliwka. 2005. "Envy and Compassion in Tournaments." Journal of Economics and Management Strategy 14 (1): 187-207.

Karni, Edi, Tim Salmon, and Barry Sopher. 2008. "Individual Sense of Fairness: An Experimental Study." Experimental Economics 11 (2): 174-89.

Kircher, Philipp, Sandra Ludwig, and Alvaro Sandroni. 2013. "On the Difference between Social and Private Goods." The B.E. Journal of Theoretical Economics 13 (1).

Machina, Mark J. 1989. "Dynamic Consistency and Non-expected Utility Models of Choice under Uncertainty." Journal of Economic Literature 27 (4): 1622-68.

Rohde, Kirsten I. M. 2010. "A Preference Foundation for Fehr and Schmidt's Model of Inequity Aversion." Social Choice and Welfare 34 (4): 537-47.

-Schmeidler, David. 1989. "Subjective Probability and Expected Utility without Additivity." Econometrica 57 (3): 571-87. 